\title{
A study on classification of EEG Data using the Filters
}

\author{
V. Baby Deepa \\ Department of Software Engineering, \\ M. Kumarasamy College of Engineernig, Karur, \\ Tamil Nadu, 639113
}

\author{
Dr. P. Thangaraj \\ Department of Computer Science \& Engineering, \\ Bannari Amman Institute of Technology, \\ Sathyamangalam, Erode, 638401
}

\begin{abstract}
In the field of data mining, classification of data is being a difficult task for further analysis. Classifying the EEG data would require more efficient algorithms. In this paper the classification filters such as Fast Hartley Transform (FHT) and Chebyshev filters are used to classify the EEG data signals. In a bulk data set of EEG signals, the signals are classified into many channels. Though various filters are available for classification, FHT with Chebyshev and FT tree only are taken to know the efficiency in classifying the EEG data signals. When these filters are applied to the data instances the percentage of correctly classified instances is high. Based on the experimental result it is suggested that these filters could be used for the enhancement of classification of EEG data.
\end{abstract}

Keywords- EEG (Electro-encephalogram); BCI (Brain Computer Interface; FHT (Fast Hartley Transform); Chebyshev filters; FT tree.

\section{INTRODUCTION}

The EEG data set obtained from BCI is used for classification. In the EEG signals there would be a cluster of features. It is vital to extract good features from that cluster. Classification of EEG dataset involves much careful effort. Identifying and extracting good features from the signals is a crucial step in the design of BCI.

The features extracted from EEG are not relevant and do not describe well the neurophysiologic signals employed, the classification algorithm which will use such features will have trouble in identifying the class of these features i.e., mental state of the user. Consequently the correct recognition rates of mental states will be very low, which will make use of the interface not convenient or even impossible for the user. It is sometimes possible to use raw signals as the input of the classification algorithm, it is recommended to select and extract good features in order to maximize the performances of the system by making easier the task of subsequent classification algorithm.

According to researchers, it seems that the choice of a good preprocessing and feature extraction method have more impact on the final performances than the choice of a good classification algorithm. In the section II the classification filters such as Fast Hartley Transform, Chebyshev filters and FT are described. This section gives a clear picture of how the classification filters function. The filters are applied to the data during the experimentation and the results are given in the section III. Finally it has been concluded that a combination of FHT, Chebyshev and FT tree have the potential to enhance the classification of EEG data.

\section{CLASSIFICATION FILTERS}

\section{A. Discrete Hartley transform (DHT)}

A Discrete Hartley transform (DHT) [1] is a Fourier-related transform of discrete, periodic data similar to the discrete Fourier transform (DFT), with analogous applications in signal processing and related fields. Its main distinction from the DFT is that it transforms real inputs to real outputs, with no intrinsic involvement of complex numbers. Because there are fast algorithms for the DHT analogous to the fast Fourier transform (FFT), the DHT was originally proposed by R. N. Bracewell in 1983 as a more efficient computational tool in the common case where the data are purely real. It was subsequently argued, however, that specialized FFT algorithms for real inputs or outputs can ordinarily be found with slightly fewer operations than any corresponding algorithm for the DHT.

\section{B. The Fast Hartley Transform (FHT)}

EEG data are inherently real valued, yet most general Fourier transform algorithms [8] accept complex valued input and return complex valued output. The generality of these algorithms is also their weakness, for in the process of transforming real data they perform twice as many operations (arithmetic, address and transfer) as is necessary. Since the Fourier transform is commonly used in the analysis of real signals, special versions of almost every transform algorithm have been developed to deal more efficiently with real data. Unfortunately, when it comes to inverse transformation, another special version of the algorithm is required to efficiently transform the complex output back into the real sequence.

The Hartley transform distinguishes itself from its close cousin, the Fourier transform [5], by being real valued; it produces real output from real input. Even so, it provides the same phase and amplitude information about the data as the Fourier transform. The Hartley transform may also be computed using a 'fast' algorithm which requires $\mathrm{O}(\mathrm{N} \log 2 \mathrm{~N})$ operations. Finally, the fast Hartley transform (FHT) $[2,3]$ is twice as fast as a complex valued FFT, requiring virtually the same number of operations as the real valued FFT algorithms. 
Formally, the discrete Hartley transform $[4,6,7]$ is a linear, invertible function $H: \mathbf{R}^{n} \rightarrow \mathbf{R}^{n}$ (where $\mathbf{R}$ denotes the set of real numbers). The $N$ real numbers $x_{0}, \ldots ., x_{N-1}$ are transformed into the $N$ real numbers $H_{0}, \ldots, H_{N-1}$ according to the formula

$$
H_{k}=\sum_{n=0}^{N-1} x_{n}\left[\cos \left(\frac{2 \pi}{N} n k\right)+\sin \left(\frac{2 \pi}{N} n k\right)\right] \quad k=0, \ldots, N-1
$$

where $\pi$ is Pi. The combination

$$
\cos (\mathrm{z})+\sin (\mathrm{z})=\sqrt{ } 2 \cos (\mathrm{z}-\pi / 4)
$$

is sometimes denoted $\cos (\mathrm{z})$, and should be contrasted with the $e^{-i z}=\cos (z)-i \sin (z)$ that appears in the DFT definition (where $i$ is the imaginary unit).

\section{Chebyshev filters}

These are analog or digital filters [9] having a steeper rolloff and more pass band ripple (type I) or stop band ripple (type II) than Butterworth filters. Chebyshev filters have the property that they minimize the error between the idealized and the actual filter characteristic over the range of the filter, but with ripples in the pass band. This type of filter is named in honor of Pafnuty Chebyshev because their mathematical characteristics are derived from Chebyshev polynomials.

Because of the passband ripple inherent in Chebyshev filters, filters which have a smoother response in the passband but a more irregular response in the stopband are preferred for some applications.

\section{1) Type I Chebyshev filter:}

These are the most common Chebyshev filters. The gain (or amplitude) response as a function of angular frequency $\omega$ of the $n$th order low pass filter is

$$
\operatorname{Gn}(\omega)=|\operatorname{Hn}(j \omega)|=\frac{1}{\sqrt{1+\varepsilon^{2}} \quad T^{2}{ }_{n}\left(\omega / \omega_{0}\right)}
$$

where $\varepsilon_{\text {is }}$ the ripple factor, $\omega_{0}$ is the cutoff frequency and $T_{n}()$ is a Chebyshev polynomial of the $n$th order.

The passband exhibits equiripple behavior, with the ripple determined by the ripple factor $\varepsilon$. In the passband, the Chebyshev polynomial alternates between 0 and 1 so the filter gain will alternate between maxima at $G=1$ and minima at $\mathrm{G}=$ $1 / \sqrt{ } 1+\varepsilon^{2}$. At the cutoff frequency $\omega_{0}$ the gain again has the value $1 / \sqrt{ } 1+\varepsilon^{2}$ but continues to drop into the stop band as the frequency increases. This behavior is shown in the diagram. The common definition of the cutoff frequency to $-3 \mathrm{~dB}$ does not hold for Chebyshev filters.

The order of a Chebyshev filter [10] is equal to the number of reactive components (for example, inductors) needed to realize the filter using analog electronics.

The ripple is often given in $\mathrm{dB}$ :

$$
\text { Ripple in } \mathrm{dB}=20 \log _{10} \quad 1
$$

so that a ripple amplitude of $3 \mathrm{~dB}$ results from $\varepsilon=1$

An even steeper roll-off can be obtained if we allow for ripple in the stop band, by allowing zeroes on the $j \omega$-axis in the complex plane. This will however result in less suppression in the stop band. The result is called an elliptic filter, also known as Cauer filters.

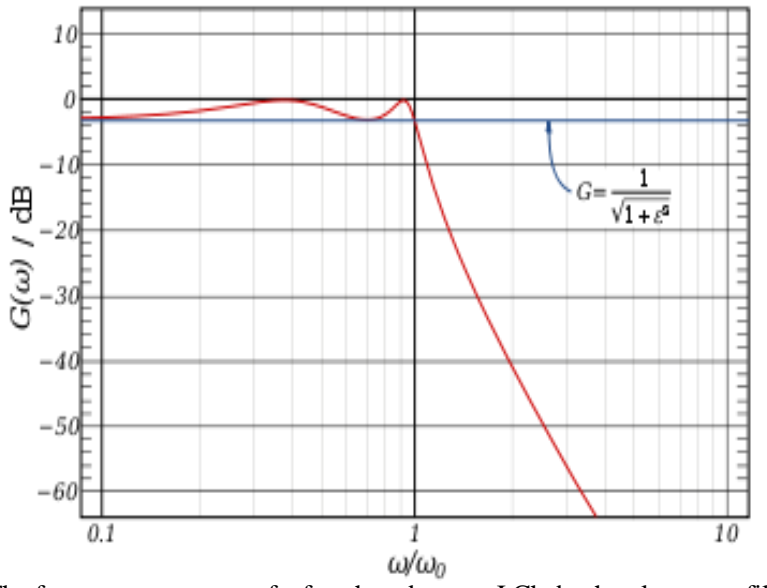

The frequency response of a fourth-order type I Chebyshev low-pass filter with $\varepsilon=1$.

\section{2) Type II Chebyshev Filter:}

This is also known as inverse Chebyshev, this type is less common because it does not roll off as fast as type I, and requires more components. It has no ripple in the passband, but does have equiripple in the stopband. The gain is:

$$
G_{n}\left(\omega, \omega_{0}\right)=\frac{1}{\sqrt{1+\frac{1}{\varepsilon^{2} T_{n}^{2}\left(\omega_{0} / \omega\right)}}} .
$$

In the stop band, the Chebyshev polynomial will oscillate between 0 and 1 so that the gain will oscillate between zero and 1

$\sqrt{1+1 / \varepsilon^{2}}$

and the smallest frequency at which this maximum is attained will be the cutoff frequency $\omega_{0}$. The parameter $\varepsilon$ is thus related to the stopband attenuation $\gamma$ in decibels by:

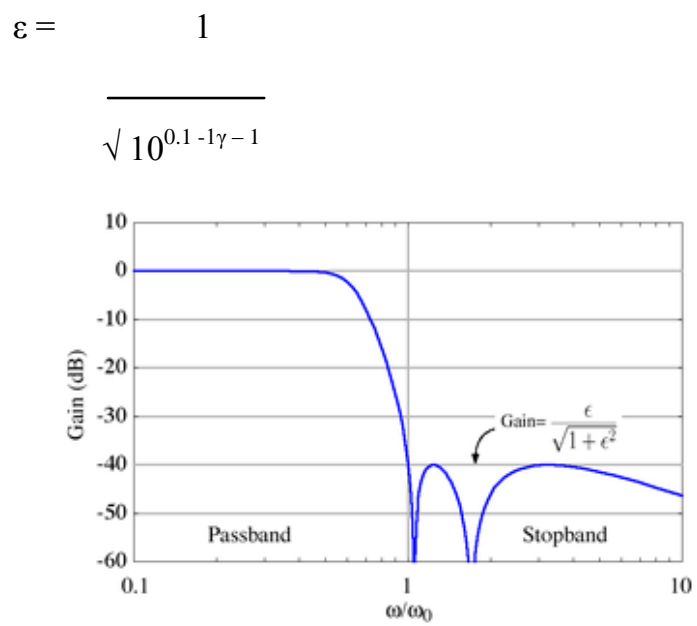

The frequency response of a fifth-order type II Chebyshev low-pass filter with $\varepsilon=0.01$ 


\section{EXPERIMENTAL RESULTS}

FHT with Chebyshev filter and FT tree classification

$===$ Stratified cross-validation $===$

$===$ Summary $===$

Correctly Classified Instances

90

Incorrectly Classified Instances

78

$53.5 \%$

Kappa statistic

$46.4 \%$

0.0672

0.4713

0.6592

Root mean squared error

$94.458 \%$

Relative absolute error

Root relative squared error

Total Number of Instances

$131.9763 \%$

\section{$===$ Confusion Matrix $===$}

a $b<--$ classified as

$3941 \mid \mathrm{a}=$ hand

$3751 \mid b=$ foot

\section{CONCLUSION}

Based on the experimental results it is concluded that while classification is done on the EEG dataset, it is suggested that the classification filters such as Fast Hartley Transform (FHT) and Chebyshev filters may be used for the better classification which may make researchers do further analysis with utmost confidence.

\section{REFERENCES}

[1] R. N. Bracewell, "Discrete Hartley transform," J. Opt. Soc. Am. 73 (12), 1832-1835 (1983).

[2] R. N. Bracewell, "The fast Hartley transform," Proc. IEEE 72 (8), 10101018 (1984).

[3] R. N. Bracewell, The Hartley Transform (Oxford Univ. Press, New York, 1986).

[4] R. N. Bracewell, "Computing with the Hartley Transform," Computers in Physics 9 (4), 373-379 (1995).

[5] R. V. L. Hartley, "A more symmetrical Fourier analysis applied to transmission problems," Proc. IRE 30, 144-150 (1942).

[6] H. V. Sorensen, D. L. Jones, C. S. Burrus, and M. T. Heideman, "On computing the discrete Hartley transform," IEEE Trans.

Acoust. Speech Sig. Processing ASSP-33 (4), 1231-1238 (1985).

[7] H. V. Sorensen, D. L. Jones, M. T. Heideman, and C. S. Burrus, "Realvalued fast Fourier transform algorithms," IEEE Trans.

Acoust. Speech Sig. Processing ASSP-35 (6), 849-863 (1987).

[8] Miodrag Popović and Dragutin Šević, "A new look at the comparison of the fast Hartley and Fourier transforms," IEEE

Trans. Signal Processing 42 (8), 2178-2182 (1994).

[9] Pierre Duhamel and Martin Vetterli, "Improved Fourier and Hartley transform algorithms: application to cyclic convolution Of real data," IEEE Trans. Acoust. Speech Sig. Processing

ASSP-35, 818-824 (1987)

[10] Daniels, Richard W. (1974). Approximation Methods for Electronic Filter Design. New York: McGraw-Hill. ISBN 0-07-015308-6.

[11] Williams, Arthur B.; Taylors, Fred J. (1988). Electronic Filter Design Handbook. New York: McGraw-Hill. ISBN 0-07-070434-1

[12] Wavelet Time-frequency Analysis of Electro-encephalogram ( EEG ) Processing. (2010). International Journal of Advanced Computer Science and Applications - IJACSA, 1(5), 1-5.

[13] Karyati, C. M., \& Muslim, A. (2011). Effect of Thrombi on Blood Flow Velocity in Small Abdominal Aortic Aneurysms from MRI Examination. International Journal of Advanced Computer Science and Applications - IJACSA, 2(3), 13-18.

\section{AUTHORS PROFILE}

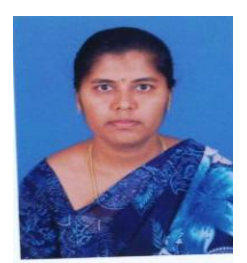

V.Baby Deepa, received her Bachelor's and Master's in Computer Science from Barathidasan University, Trichy and did her M.Phil. as well in the same university. She has 12 years of teaching experience and is Assistant professor in the faculty of Software Engineering, she is serving as the head for the same faculty in M.Kumarasamy College of Engineering, Karur. She has presented more than 15 papers on various topics including national, international conference and journals. She is a research scholar of Anna University Chennai and her research area is Fuzzy and Data Mining.

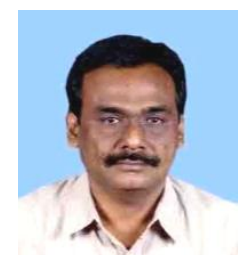

Dr.P.Thangaraj, did his graduation and post graduation in Mathematics at Madras University.He completed his M.Phil degree in the year 1993 from Bharathiar University. He completed his research work on Fuzzy Metric Spaces and awarded Ph.D degree by Bharathiar University.He completed the post graduation in Computer Applications at ICNOU in 2005. He completed his Master of Engineering degree in Computer Science in the year 2007 at Vinayaka Missions University. Currently he is a professor and Head of Computer Science and Engineering in Bannariamman College of Engineering and Technology. His current area of research interest is in Fuzzy Metric Spaces and Data Mining. 